\title{
A Systematic Review and Meta-Analysis on the Association between Inflammatory Bowel Disease Family History and Colorectal Cancer
}

\author{
Hadis Najafimehr, ${ }^{1}$ Hamid Asadzadeh Aghdaei, ${ }^{1,2}$ Mohamad Amin Pourhoseingholi ${ }^{1}{ }^{2}$ \\ Hamid Mohaghegh Shalmani, ${ }^{1}$ Amir Vahedian-Azimi $\odot{ }^{3}{ }^{3}$ Matthew Kroh, ${ }^{4}$ \\ Mohammad Reza Zali, ${ }^{2}$ and Amirhossein Sahebkar $\mathbb{D}^{5,6,7}$ \\ ${ }^{1}$ Basic and Molecular Epidemiology of Gastrointestinal Disorders Research Center, Research Institute for Gastroenterology and \\ Liver Diseases, Shahid Beheshti University of Medical Sciences, Tehran, Iran \\ ${ }^{2}$ Gastroenterology and Liver Diseases Research Center, Research Institute for Gastroenterology and Liver Diseases, Shahid Beheshti \\ University of Medical Sciences, Tehran, Iran \\ ${ }^{3}$ Student Research Committee, Hamadan University of Medical Sciences, Hamadan, Iran \\ ${ }^{4}$ Digestive Disease and Surgery Institute, Cleveland Clinic Lerner College of Medicine, Cleveland, OH, USA \\ ${ }^{5}$ Biotechnology Research Center, Pharmaceutical Technology Institute, Mashhad University of Medical Sciences, Mashhad, Iran \\ ${ }^{6}$ Applied Biomedical Research Center, Mashhad University of Medical Sciences, Mashhad, Iran \\ ${ }^{7}$ School of Pharmacy, Mashhad University of Medical Sciences, Mashhad, Iran
}

Correspondence should be addressed to Mohamad Amin Pourhoseingholi; aminphg@gmail.com and Amirhossein Sahebkar; amir_saheb2000@yahoo.com

Received 28 June 2021; Accepted 8 October 2021; Published 23 October 2021

Academic Editor: Muhammad Naeem

Copyright (c) 2021 Hadis Najafimehr et al. This is an open access article distributed under the Creative Commons Attribution License, which permits unrestricted use, distribution, and reproduction in any medium, provided the original work is properly cited.

Background. Colorectal cancer (CRC) and inflammatory bowel disease (IBD) are closely interrelated. However, the effect of having a family history of one disease on the risk of another remains undetermined. Aim. The purpose of this meta-analysis was to estimate the prevalence of a family history of CRC among patients with IBD, as well as the prevalence of a family history of IBD among patients with CRC. Methods. PubMed, Scopus, Embase, Web of Science, and Google Scholar were searched to identify studies reporting the prevalence of family history of IBD among patients with CRC, in addition to the prevalence of family history of CRC among IBD patients. Criteria for study inclusion consisted of the following: (1) studies that evaluated either IBD or CRC and dysplasia, (2) included all age groups, and (3) evaluated the family history effects for IBD or CRC. The total number of IBD patients and IBD patients with a family history of CRC and the total number of CRC patients and CRC patients with a family history of IBD were reviewed. The pooled prevalence of diseases was also estimated according to degree of relatives and geographical area. Random-effects models were used for estimating pooled prevalence. Results. A total of 27 studies were included with 26,576 IBD and 9,181 CRC or dysplasia patients. Eligible studies included 13 case-control, 10 cohort, and 4 cross-sectional types. The pooled prevalence of a family history of CRC among patients with IBD was 6\% (95\% CI: 4-9\%). The pooled prevalence for first- and second-degree relatives (11\%, 95\% CI: $0-37 \%)$ was more than that for the other relative subgroups of relatedness degree. The prevalence in the American regions $(8 \%(95 \%$ CI: $5-13 \%))$ was higher than that in the others. The pooled prevalence for a family history of IBD among CRC or dysplasia patients was $11 \%$ (95\% CI: 6-16\%). The pooled prevalence for first-degree relatives (13\% (95\% CI: 3-28\%) was higher than that for the other relative subgroups of relatedness degree; it was also greater in American countries (15\%, 95\% CI: 8-23\%). Conclusion. This study emphasizes the relationship between a family history of IBD and CRC development. Additionally, there was notable prevalence for a family history of CRC among IBD patients. American countries and first-degree relatives were identified to have a higher prevalence for both disease processes. 


\section{Introduction}

Colorectal cancer (CRC) is one of the most common cancers worldwide [1]. Familial studies have demonstrated that having a family history of CRC may increase an individual's risk of developing CRC and that this risk in individuals with a first-degree relative with $\mathrm{CRC}$ is more than 2 -folds greater $[2,3]$. One of the most important risk factors of CRC is inflammatory bowel disease (IBD). IBD is an immunemediated gastrointestinal disorder that is identified with subtypes of Crohn's disease (CD) and ulcerative colitis (UC) [4]. Both CD and UC patients are at risk of CRC development [5]. Like CRC, family history is one of the strongest risk factors for development of IBD [6].

It has been shown that CRC is a relatively common and life-threatening consequence of IBD, especially UC. This is likely secondary to proneoplastic effects of chronic intestinal inflammation. Duration, extent and severity of IBD, the existence of inflammatory pseudopolyps, presence of primary sclerosing cholangitis, and a family history of CRC are the main risk factors of IBD-related CRC [7]. A family history of CRC independently increases CRC risk two- to threefolds in patients with UC (OR: 3.7, 95\% CI: 1.0-13.2) [8].

There are common factors inducing the development of IBD and CRC, such as the variations in gut microbiota and in the interleukin pathways and tumour necrosis factor, as well as also age, race, genetics, family history, diet composition, obesity, and vitamin and mineral levels [9]. Moreover, it is shown that IBD-related CRC patients are younger and have high prevalence of multiple cancerous lesions [10]. This suggests that in addition to inflammation, other factors may be involved in the pathogenesis of IBD-related CRC.

To determine a quantitative data for the prevalence of a family history of CRC or IBD, there are only a few comprehensive studies. In a previous meta-analysis, the prevalence of CRC in patients with UC has been estimated at 3.7\%, across the world [11]. In a study by Shi et al., the prevalence of a family history of IBD among groups of Caucasians, Asians, Blacks, and Hispanics has been estimated at $12 \%$, $0.04 \%, 0.07 \%$, and $0.13 \%$, respectively [12]. In the other study by Childers et al., it was revealed that the prevalence of a family history of IBD among patients with UC is $12 \%$ (range: 0-39\%) [13].

Despite CRC and IBD being closely interrelated, the relation between a family history of each disease and the risk of developing the other still has not been quantified. To address this gap, we performed a systematic review and metaanalysis for estimation of the prevalence of a family history of CRC among patients with IBD as well as the prevalence of a family history of IBD among patients with CRC.

\section{Methods}

2.1. Search Strategy. Our electronic search was limited to the English language, and it was conducted in PubMed, Scopus, Embase, Web of Science, and Google Scholar by using the following keywords: ("inflammatory bowel disease" or "ulcerative colitis" or "crohn's disease") and ("colorectal cancer" or "colon and rectum cancer" or "dysplasia or neo- plasia") and ("family history" or "relative" or "familial"). Published studies up to December 2020 were considered, and references of individual studies were searched to find other eligible studies. The Preferred Reporting Items for Systematic review and Meta-Analysis (PRISMA) guideline was used for reporting this study [14].

2.2. Inclusion and Exclusion Criteria. The authors reviewed titles and abstracts of original full-text articles performed on each of the IBD or CRC patients. The inclusion criteria were as follows: (1) studies that evaluated either IBD or CRC and dysplasia, (2) included all age groups, and (3) evaluated the family history effects for IBD or CRC. The exclusion criteria were as follows: (1) studies with an unknown number of patients with a family history for IBD or CRC and (2) conducted on animals (mice). The authors excluded all reviews or conference abstracts and non-English publications. For quality control of studies, the authors used the Newcastle-Ottawa Scale (NOS) and the high and moderate quality articles considered as eligible [15]. The disagreements among the authors on the choice of the eligible studies were discussed, and finally, any disagreement was evaluated by the senior investigator.

2.3. Data Extraction. For each selected study, the authors extracted the following information: name of the first author, year of publication, country of publication, total sample size, study design, total number of IBD patients and IBD patients with family history of CRC, total number of CRC patients and CRC patients with family history of IBD, and degree of relatives included.

2.4. Outcome of Interest. The main outcomes of this metaanalysis were the prevalence of a family history of IBD in $\mathrm{CRC}$ as well as the prevalence of a family history of CRC in IBD.

2.5. Statistical Analysis. All analyses were done using Stata 14 software, and 0.05 was considered as the statistical significance level. For each outcome of interest, the corresponding proportion was calculated via the extracted data from each eligible study. Pooled prevalence with $95 \%$ confidence interval (CI) was estimated using the random-effects model wherever the prevalence has been reported. In the process of prevalence merging, the outcomes with zero event were adjusted using the "Freeman-Tukey double arcsine" transformation in the "metaprop" procedure [16]. The heterogeneity was evaluated by using the Cochran's $Q$ test and $I^{2}$ statistic and $P$ value. We performed stratified analysis for items that may cause heterogeneity. Publication bias was examined by using Begg's and Egger's tests [17] and also funnel plot [18].

\section{Results}

3.1. Process of Study Selection. After a comprehensive search of the databases, 131 studies were obtained. We excluded 40 studies after examining the title and abstract. The number of studies selected for primary evaluation was 91 . Then, 53 studies were excluded because they did not meet inclusion 


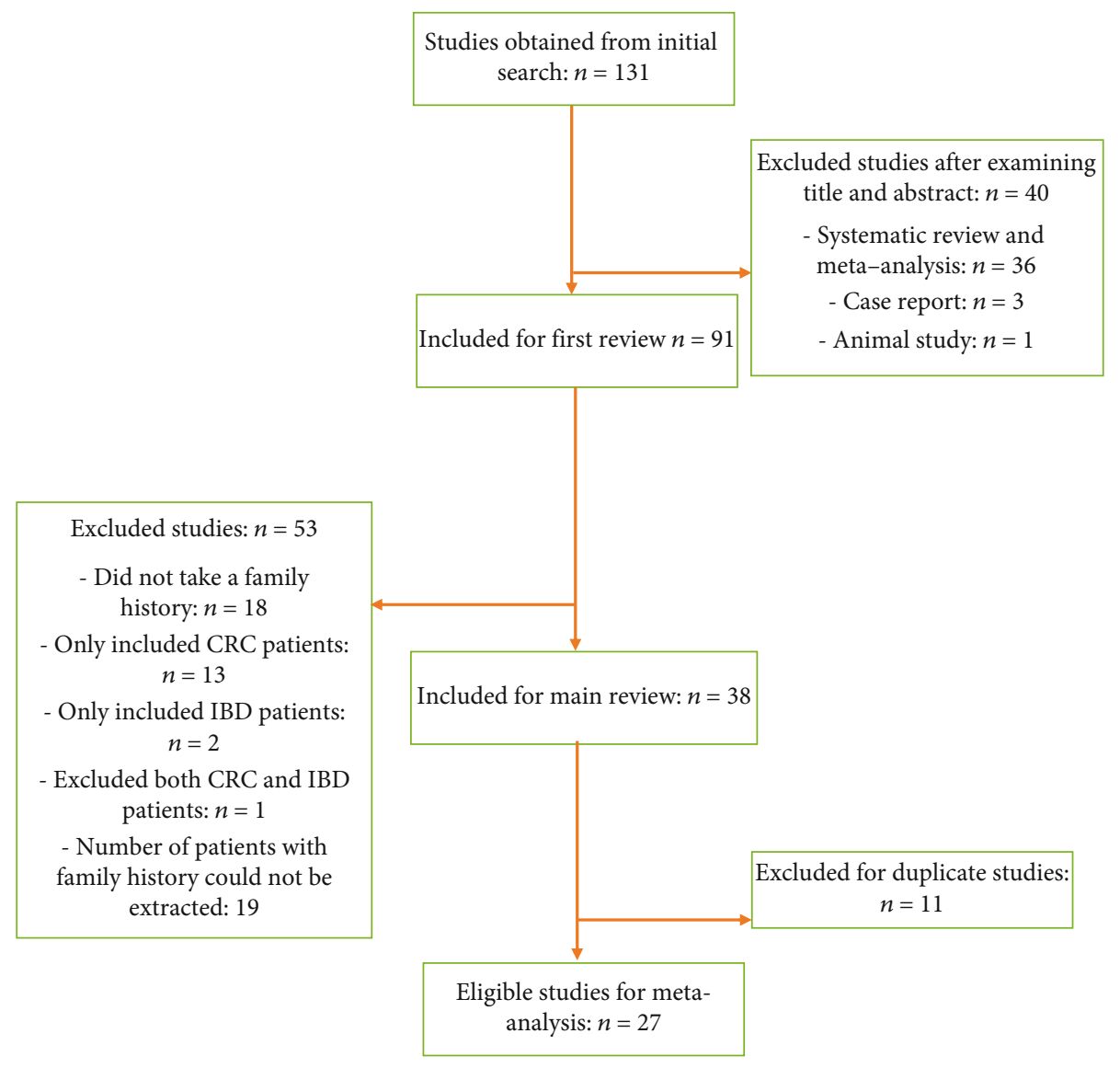

Figure 1: Flow chart for the process of study selection.

criteria or did not report all necessary information. There were 11 republished studies that contained duplicate data samples which were excluded. Finally, 27 studies were considered as eligible and enrolled in the meta-analysis. The details of study selection are presented in Figure 1.

3.2. Characteristics of the Eligible Studies. Eligible studies included 13 case-control, 10 cohort, and 4 cross-sectional types [8, 19-44]. The interest disease which was considered in most studies was IBD-related CRC, and the main target was often evaluation of the factor associated with CRC. Some cohort studies such as Brackmann et al. followed up patients with IBD and evaluated the influence of family history of CRC on survival [19]. Other cohort types including Askling et al.'s study followed up UC and CD patients, and they concluded that having both family histories of IBD and CRC increases the risk of CRC [20]. In some of the case-control studies, the main aim was to review supplementation (aminosalicylate and folic acid) effect on the risk of IBD or CRC $[21,22]$. The characteristics of the eligible studies are presented in Table 1. The number of IBD and CRC or dysplasia patients represented by 27 eligible studies was 26,576 and 9,181, respectively. All studies except three reported the age at IBD diagnosis. For IBD patients, the mean age at IBD diagnosis was $34.52 \pm 7.79$ years (range: 25-29), and for IBD patients with CRC or dysplasia, this mean was $35.35 \pm 8.65$ years (range: $25-57.4$ ).
3.3. Family History of CRC among IBD Patients. There were 26 studies on the family history of CRC, including 25,819 IBD patients. The pooled overall prevalence of a family history of CRC among patients with IBD was 6\% (95\% CI: 4$9 \%, P<0.001)$ with $I^{2}=96.01 \%, P<0.001$. The forest plot of the result is presented in Figure 2(a).

3.3.1. Family History of CRC among IBD Patients by Degree of Relative. The degrees of relatives in the extracted studies were reported as first (including 9 studies with 2,357 IBD patients), first and second (including 3 studies with 594 IBD patients), all degrees (including 5 studies with 22,316 IBD patients), and not reported degree (including 5 studies with 492 IBD patients). More studies were conducted on first-degree relatives, while there were studies that did not report any degree of family connection. The pooled prevalence of a family history of CRC among patients with IBD for first- and second-degree relatives (11\%, 95\% CI: $0-37$ ) was more than any other degree of relatedness (Figure 2(b)).

3.3.2. Family History of CRC among IBD Patients by Region of Study. The studies were conducted in the regions of the Americas (including the USA with 1,419 IBD patients), Europe (including Sweden, France, Netherlands, England, Portugal, and Norway with 24,279 IBD patients), and Asia (including Japan and India with 89 IBD patients). The pooled prevalence of a family history of CRC among patients 


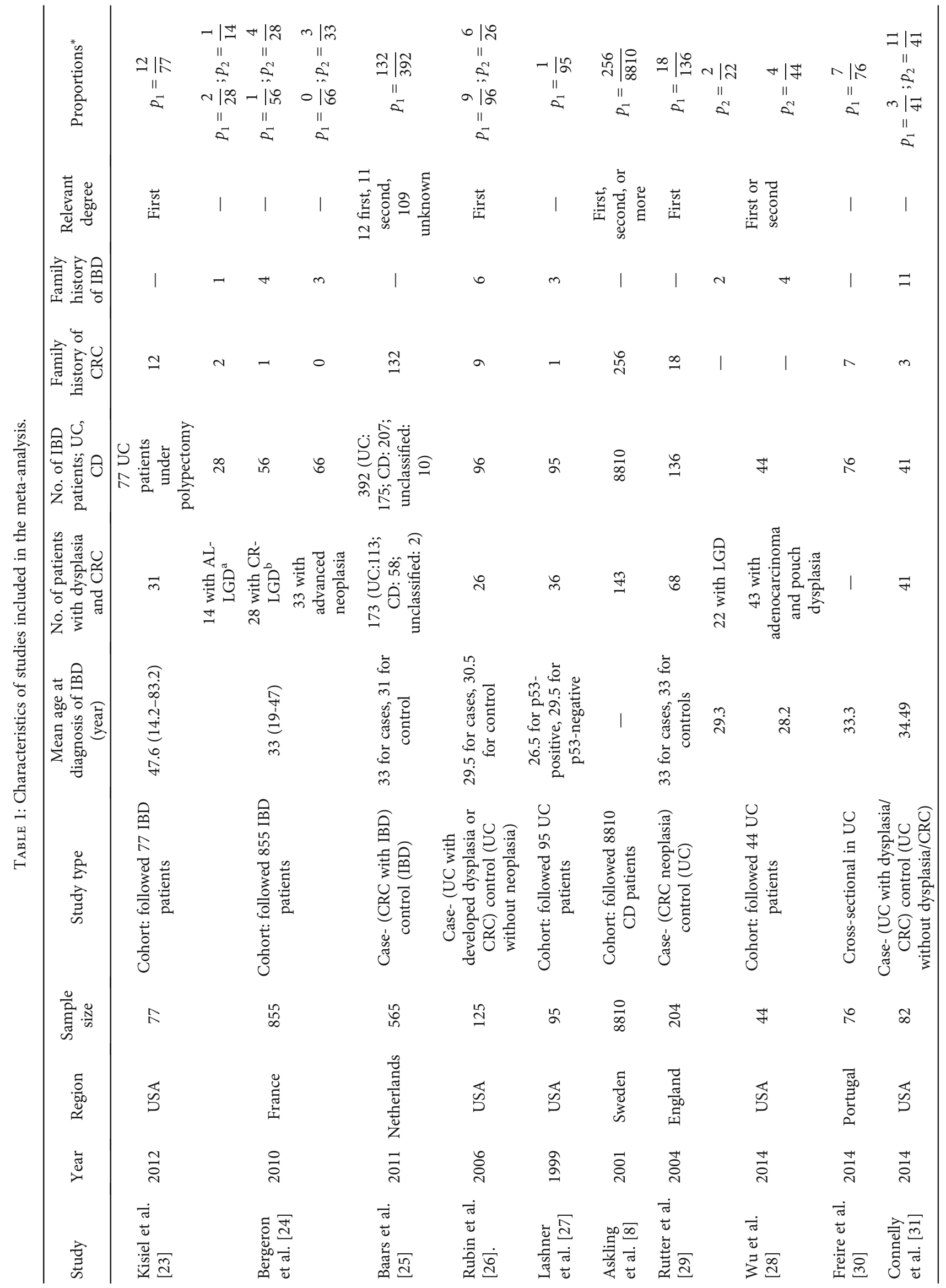




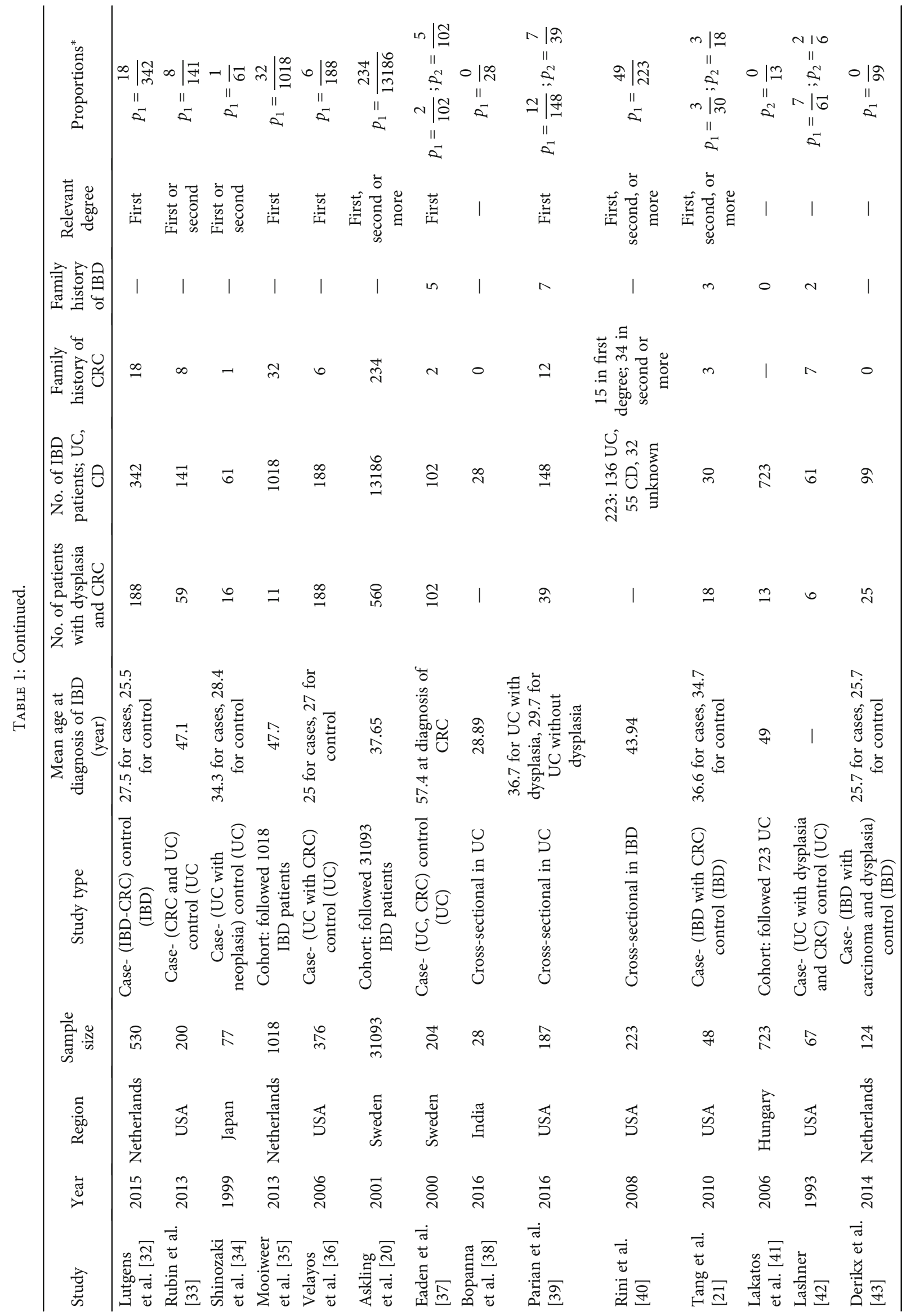




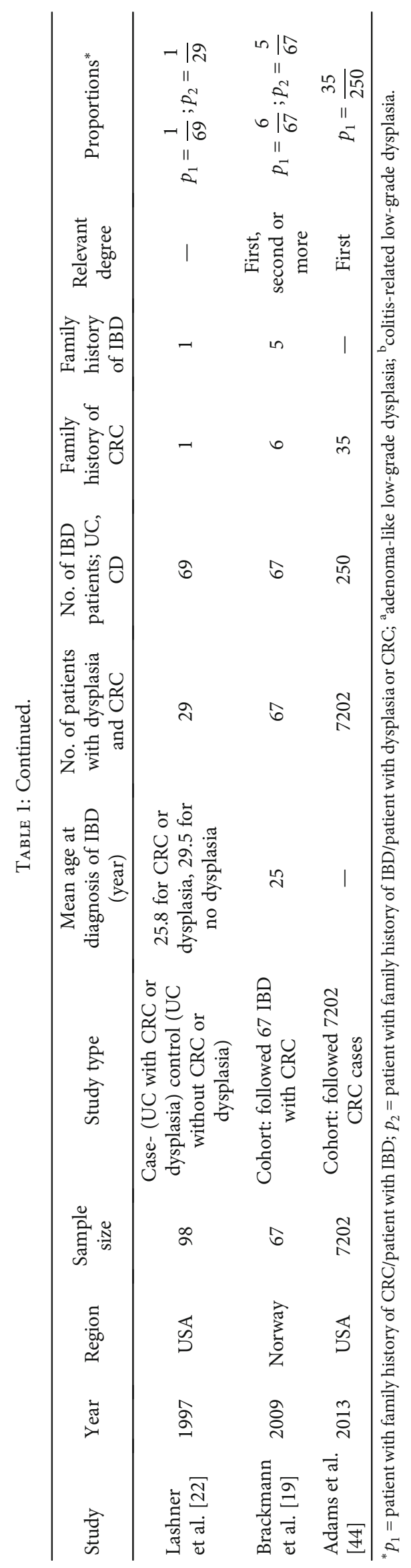




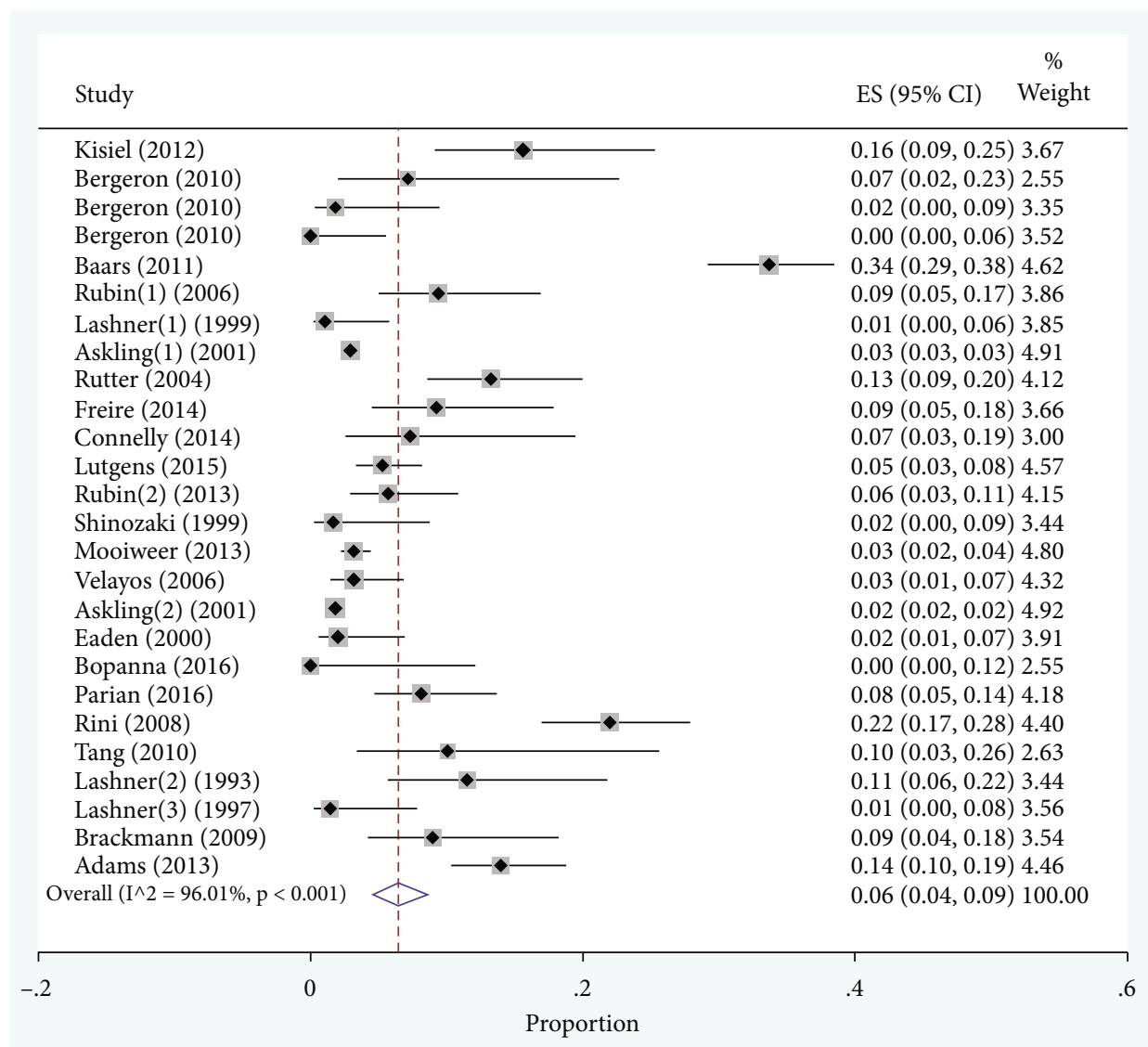

(a)

Figure 2: Continued. 


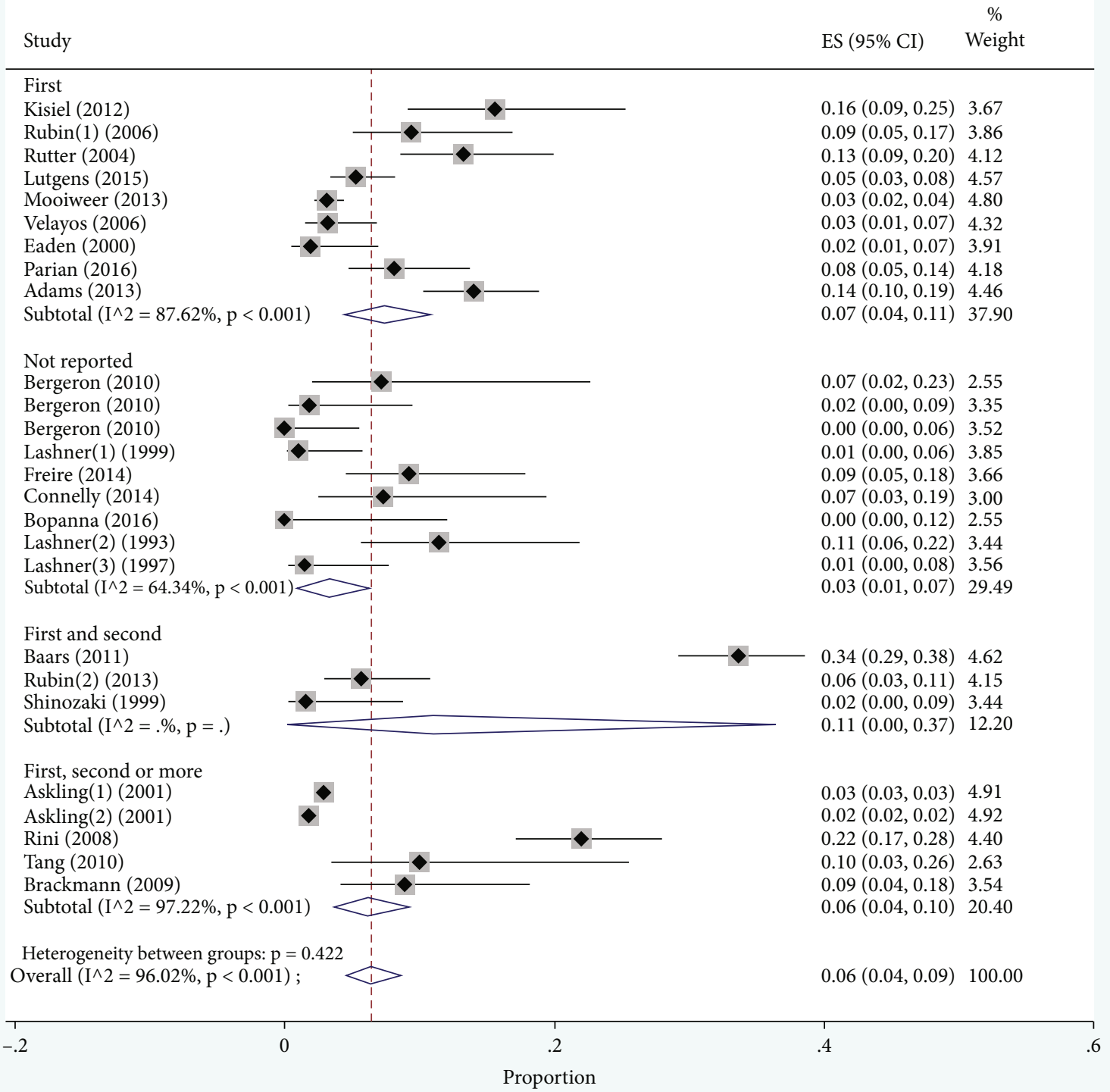

(b)

FIgURE 2: Continued. 


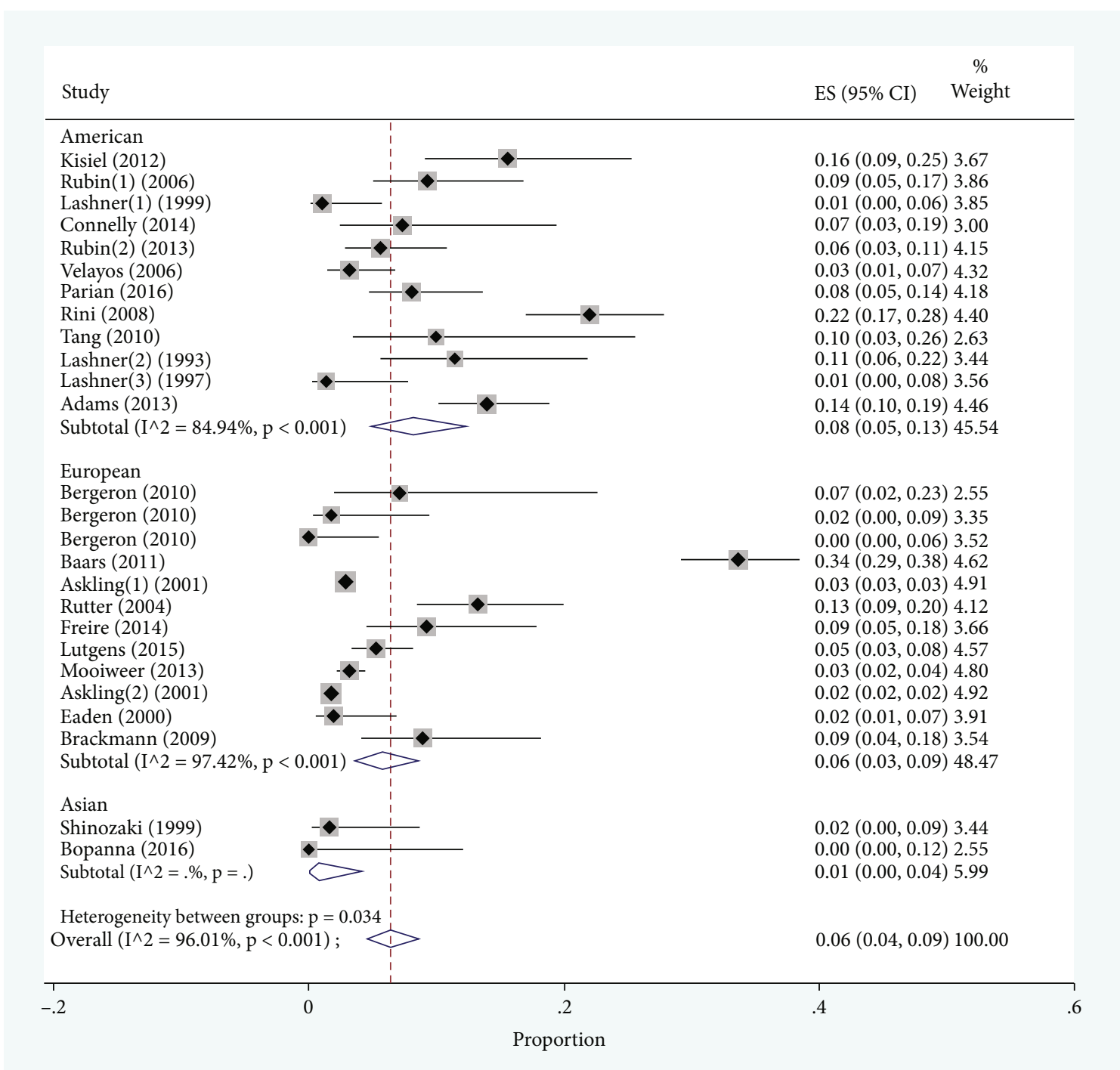

(c)

FIGURE 2: Forest plot for the prevalence of family history of CRC among IBD patients: (a) overall prevalence; (b) prevalence by degree of relative; (c) prevalence by region of study.

with IBD in the American regions (8\% (95\% CI: 5-13\%)) was higher than that in the others (Figure 2(c)).

3.4. Family History of IBD among Patients with Dysplasia or $C R C$. The number of studies concerning family history of IBD for CRC patients was 10, including 481 patients with dysplasia or CRC. The pooled prevalence for a family history of IBD among CRC or dysplasia patients was $11 \%$ (95\% CI: $6-16 \%, P<0.001)$ with $I^{2}=54.57 \%, P=0.01$. The forest plot is shown in Figure 3(a).

3.4.1. Family History of IBD among Patients with Dysplasia or CRC by Degree of Relative. First degree (including 167 patients with dysplasia or CRC), first and second degrees (including 65 patients with dysplasia or CRC), and first, second, and more degrees (including 85 patients with dysplasia or CRC) were among the reported degree of relatives in the eligible studies. Also, some studies did not report the degree (including 164 patients). The pooled prevalence in the first degree (13\% (95\% CI: 3-28\%) was higher than that in the other groups (Figure 3(b)).

3.4.2. Family History of IBD among Patients with Dysplasia or CRC by Region of Study. The regions of the Americas (including the USA, with 224 dysplasia or CRC patients) and Europe (including France, Norway, Hungary, and Sweden with 257 dysplasia or CRC patients) were the areas represented in the studies reviewed. The pooled prevalence for American countries (15\%, 95\% CI: 8-23\%) was greater than that for the European region (Figure 3(c)).

3.5. Evaluation of Publication Bias. The results of Egger's $(P=0.08)$ and Begg's $(P=0.48)$ tests revealed that there is no publication bias among the studies from which the prevalence of family history of CRC was. Also, for family history of IBD, the tests of Egger $(P=0.78)$ and Begg $(P=0.32)$ 


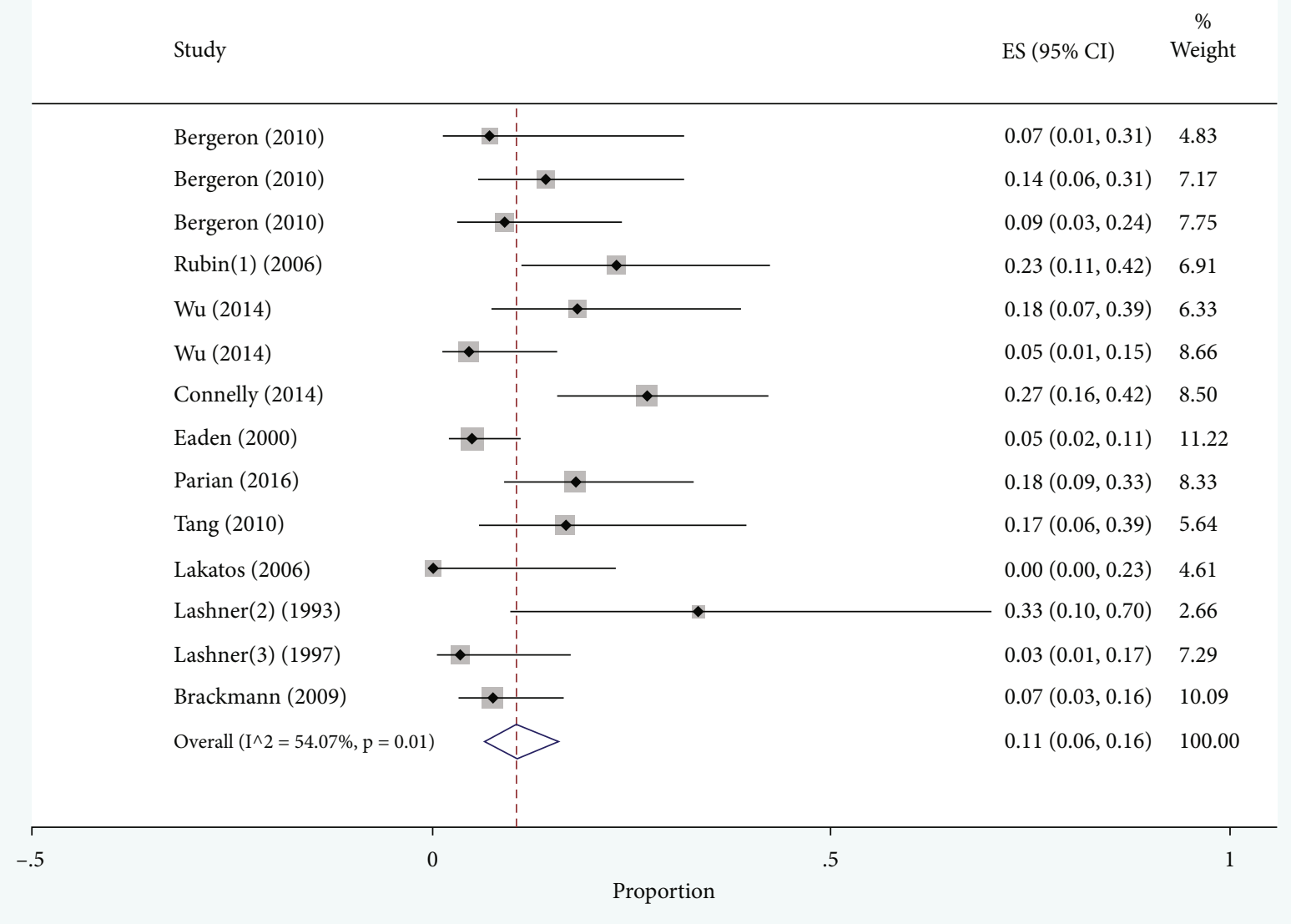

(a)

Figure 3: Continued. 


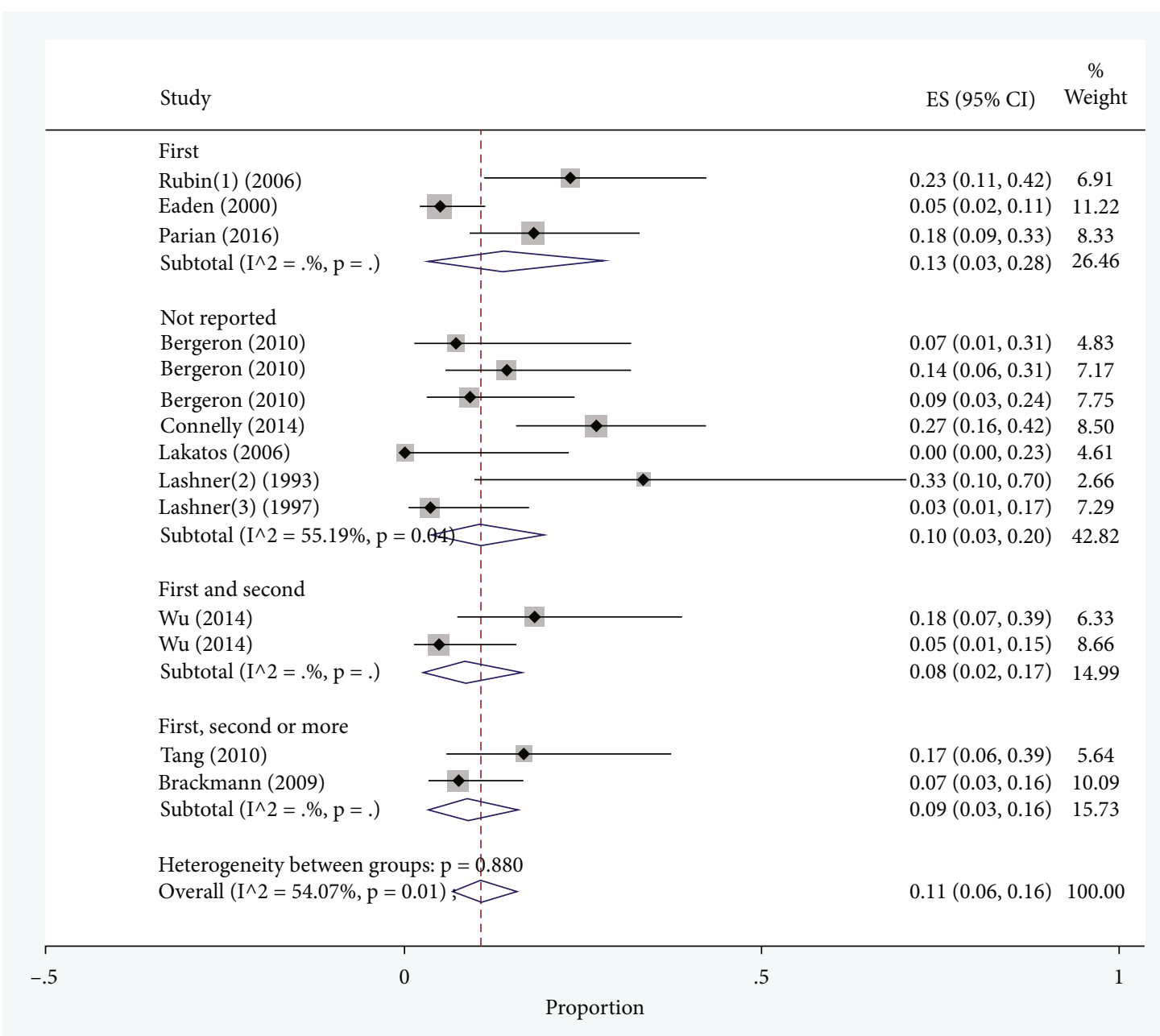

(b)

FIgUre 3: Continued. 


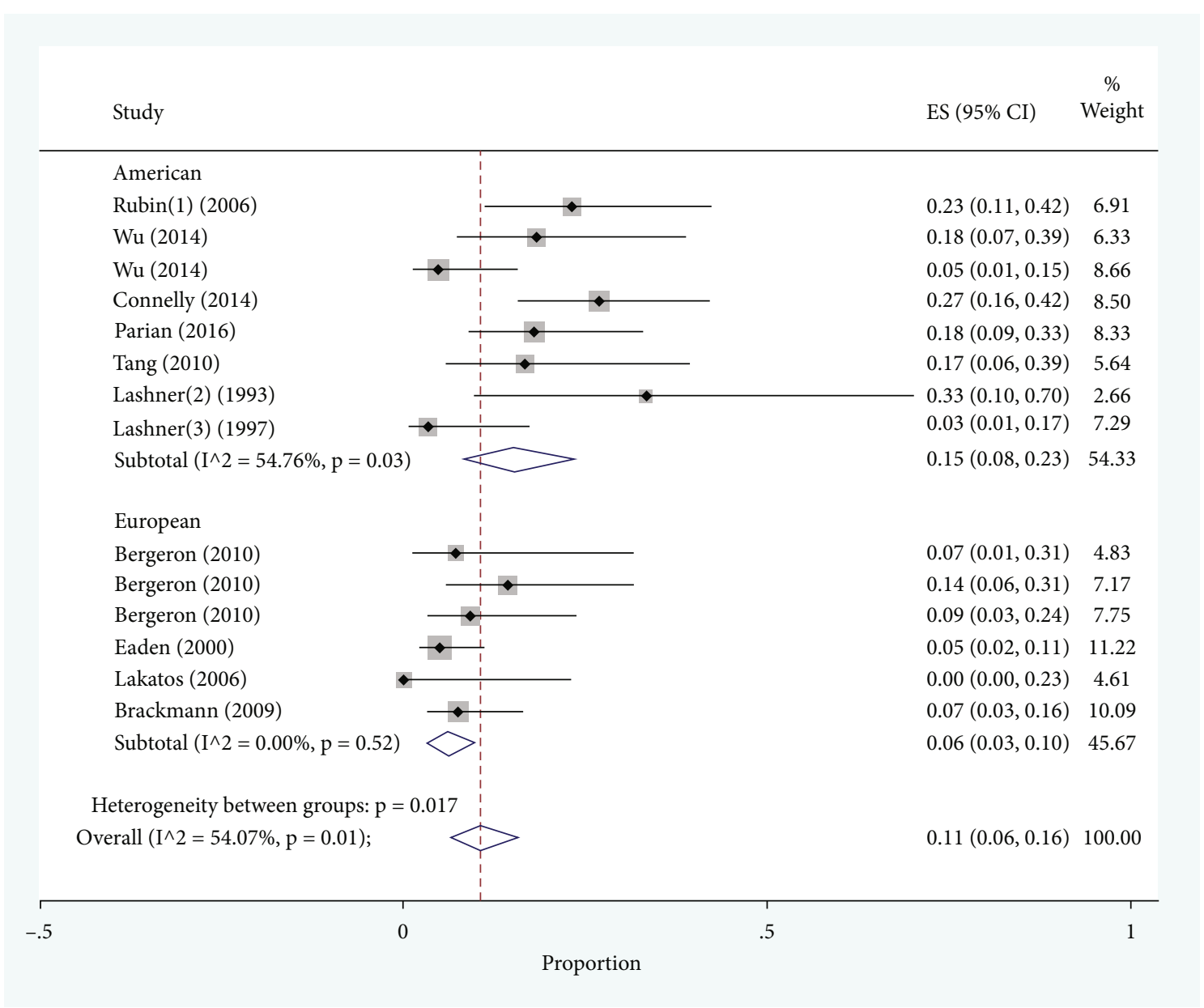

(c)

FIGURE 3: Forest plot for the prevalence of family history of IBD among dysplasia or CRC patients: (a) overall prevalence; (b) prevalence by degree of relative; (c) prevalence by region of study.

showed similar results. Additionally, the funnel plots showed evidence of an approximate symmetry (Figure 4).

\section{Discussion}

The present study is the first meta-analysis that estimates the prevalence of a family history of CRC among patients with IBD. Interestingly, we found that among IBD patients, the prevalence of a family history of CRC was 6\% (95\% CI: 49). Additionally, the pooled prevalence of a family history of IBD among patients with CRC or dysplasia was estimated to be $11 \%$ (95\% CI: 6-16). The reason for the greater latter prevalence may be that previous cohort studies proved that the history of IBD is a factor associated with CRC and the probability of developing CRC for IBD patients in the future is 2-folds higher compared with that for others [45-47]. It is important to note that IBD patients with longer duration and extensive disease and patients with diagnosis at young age are at higher risk of CRC [48]. Among IBD patients, also, the prevalence of a family history of CRC may be notable and the present study confirmed this.

In a recent meta-analysis on the influence of ethnicity in IBD prevalence by Shi et al., the mean age of IBD diagnosis was reported as 30 years [12]. This mean age in other epidemiological studies has varied, with reports of 32.7 [49], 38.46 [50], and 54.1 [51] years of age. These differences may be due to differences in access to health care center for diagnosis and overall awareness about IBD. In our study, we observed that the mean age of IBD diagnosis for IBD patients was close to 34.52 (range: 25-29), and for IBD patients with CRC, this was slightly later (35.35, range: 25 57.4). This may mean that at a later mean age of diagnosis, patients with IBD are at higher risk for CRC than young people $[52,53]$.

In our study, the authors reported the pooled prevalence of family history of CRC among patients with IBD as well as a pooled prevalence of a family history of IBD among patients with CRC or dysplasia, according to the degree of relatedness. The result demonstrated that the prevalence of family history for first- or first- and second-degree relationships is greater than that for other degrees. Previous studies revealed that the risk of gastrointestinal cancers for individuals with affected family members, especially for first-degree family members, is high and our result is in line with this point $[20,54]$.

Considering the geographical aspect, the present metaanalysis revealed that the pooled prevalence of family history 


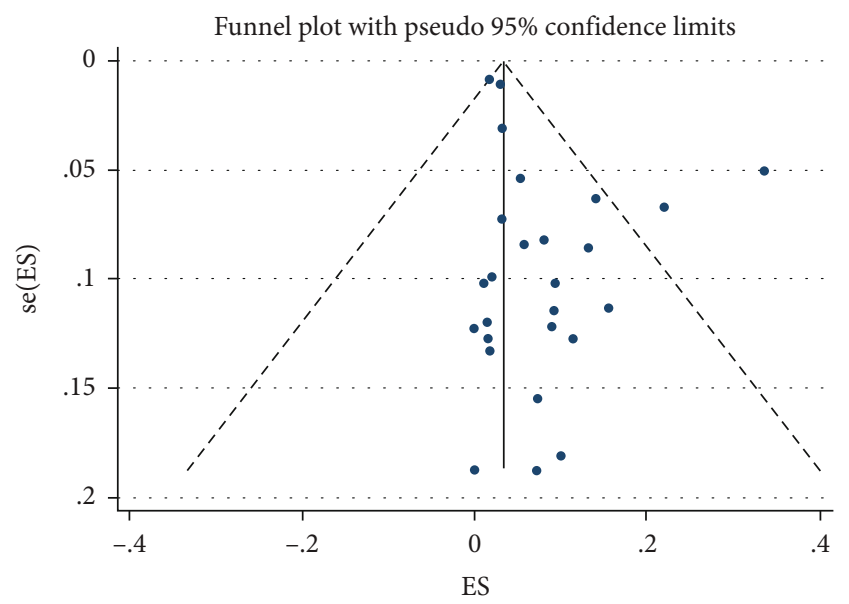

(a)

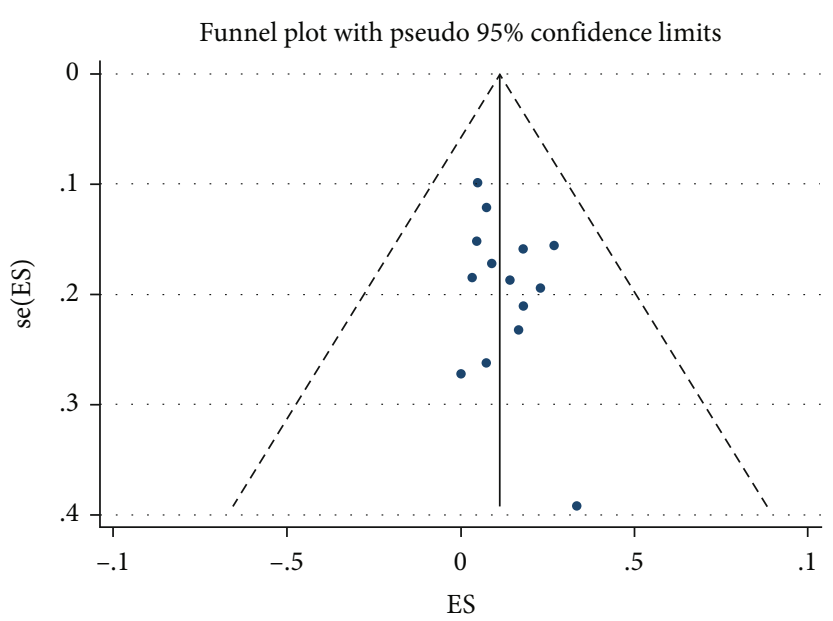

(b)

FIGURE 4: Funnel plot for assessing publication bias in the eligible studies: (a) studies provided data on family history of CRC; (b) studies provided data on family history of IBD.

of either IBD or CRC among Americans was more than that among European and Asian countries. Perhaps the global cancer statistics are helpful for finding the reason: the incidence of CRC is more common in more highly developed countries. The CRC incidence rate in Europe and Northern America is highest in comparison with that in other regions. The reason is that the prevalence of CRC risk factors including obesity and unhealthy diet in these regions is high [55, 56]. Additionally, global comparison for the prevalence of IBD has shown the highest prevalence in European and American areas and the prevalence has remained higher up until 2018 [57].

For IBD, diagnosis and management are complex and utilize clinical presentation, biomarkers, and pathology. Patient manifestation of symptoms may be due to genetic, environmental factors, and possibly molecular mechanisms within the gut microbiota patterns. All of these areas may be targets for personalized IBD treatment. This tailored approach is important for early diagnosis and treatment in the IBD management $[58,59]$. Early IBD diagnosis and successful treatment may result in decreased rates or prevention of CRC.

Previous studies observed significant heterogeneity among the results of studies. The heterogeneity is a phenomenon that usually is seen in the meta-analyses of proportion. Instead, we performed a subgroup analysis to create some more homogeneous groups of studies. But the subgroup analysis is performed by dividing studies into stratum, and this may not be useful in all cases. Generally, the more likely cause of heterogeneity, in addition to measurement errors, may be due to the way of constructing the study, including methods as well as differences in the span of the definitions [60].

There are some limitations in the present meta-analysis. First, there is overall a lack of determining the number of patients with affected family member for subtypes of UC or $\mathrm{CD}$ in the eligible studies. This limitation caused the authors to not take the two main types of pooled prevalence for each subtype. Second, some eligible studies did not report the degree of relatedness for affected family members. Also, for first-degree relatives, the type of relative (parent or sibling) was not mentioned. With more complete data, the results could be expanded. Further genetic studies are needed to determine the number of subjects with family affected member for both IBD and CRC in details of IBD subtypes, as well as sex in each type.

The present study emphasized the importance of a family history of IBD (or CRC) in the possibility of the CRC onset (or IBD). The advancement of CRC in non-IBD patients, with family history of IBD, leads us to look up further probable factors that may be common among both diseases. Gut microbiota, interleukin, and tumour necrosis factor pathways, race, genetics, family history, and diets are important factors that should be considered in the future studies [9]. The prevalence of a family history of CRC among IBD patients in American and European countries and for first-degree relatives is higher. There is a similar pattern for the prevalence of a family history of IBD among dysplasia or CRC patients. Thus, knowing the prevalence of a family history component for an at-risk population may be helpful in patient's care and managing both CRC and IBD.

\section{Data Availability}

There is no raw data associated with this article.

\section{Conflicts of Interest}

All authors have no potential conflict of interest relevant to this article.

\section{Authors' Contributions}

Hamid Asadzadeh Aghdaei and Hadis Najafimehr are cofirst authors. 


\section{Acknowledgments}

This study was supported by the Gastroenterology and Liver Diseases Research Center, Research Institute for Gastroenterology and Liver Diseases, Shahid Beheshti University of Medical Sciences, Tehran, Iran.

\section{References}

[1] D. M. Parkin, F. Bray, J. Ferlay, and P. Pisani, "Estimating the world cancer burden: Globocan 2000," International Journal of Cancer, vol. 94, pp. 153-156, 2001.

[2] G. G. Kaplan and S. C. Ng, "Understanding and preventing the global increase of inflammatory bowel disease," Gastroenterology, vol. 152, pp. 313-321. e2, 2017.

[3] T. A. Ullman and S. H. Itzkowitz, "Intestinal inflammation and cancer," Gastroenterology, vol. 140, pp. 1807-1816.e1, 2011.

[4] D. A. Ouakrim, T. Lockett, A. Boussioutas, J. L. Hopper, and M. A. Jenkins, "Screening participation for people at increased risk of colorectal cancer due to family history: a systematic review and meta-analysis," Familial Cancer, vol. 12, pp. 459472, 2013.

[5] L. Baglietto, M. A. Jenkins, G. Severi et al., "Measures of familial aggregation depend on definition of family history: metaanalysis for colorectal cancer," Journal of Clinical Epidemiology, vol. 59, pp. 114-124, 2006.

[6] W. E. Ek, M. D'amato, and J. Halfvarson, "The history of genetics in inflammatory bowel disease," Annals of Gastroenterology: quarterly publication of the Hellenic Society of Gastroenterology, vol. 27, p. 294, 2014.

[7] R. W. Stidham and P. D. Higgins, "Colorectal cancer in inflammatory bowel disease," Clinics in Colon and Rectal Surgery, vol. 31, pp. 168-178, 2018.

[8] J. Askling, P. W. Dickman, A. Ekbom et al., "Family history as a risk factor for colorectal cancer in inflammatory bowel disease," Gastroenterology, vol. 120, pp. 1356-1362, 2001.

[9] M. S. Nadeem, V. Kumar, F. A. Al-Abbasi, M. A. Kamal, and F. Anwar, "Risk of colorectal cancer in inflammatory bowel diseases," Seminars in Cancer Biology, vol. 64, pp. 51-60, 2020.

[10] M. Yashiro, "Ulcerative colitis-associated colorectal cancer," World Journal of Gastroenterology: WJG, vol. 20, pp. 1638916397, 2014.

[11] J. Eaden, K. Abrams, and J. Mayberry, "The risk of colorectal cancer in ulcerative colitis: a meta-analysis," Gut, vol. 48, pp. 526-535, 2001.

[12] H. Y. Shi, A. N. Levy, H. D. Trivedi, F. K. Chan, S. C. Ng, and A. N. Ananthakrishnan, "Ethnicity influences phenotype and outcomes in inflammatory bowel disease: a systematic review and meta-analysis of population-based studies," Clinical Gastroenterology and Hepatology, vol. 16, pp. 190-197.e11, 2018.

[13] R. E. Childers, S. Eluri, C. Vazquez, R. M. Weise, T. M. Bayless, and S. Hutfless, "Family history of inflammatory bowel disease among patients with ulcerative colitis: a systematic review and meta-analysis," Journal of Crohn's and Colitis, vol. 8, pp. 14801497, 2014.

[14] D. Moher, A. Liberati, J. Tetzlaff, and D. G. Altman, "Preferred reporting items for systematic reviews and meta-analyses: the PRISMA statement," International Journal of Surgery, vol. 8, pp. 336-341, 2010.

[15] G. Wells, B. Shea, D. O'connell et al., The Newcastle-Ottawa Scale (NOS) for assessing the quality of nonrandomised studies in meta-analyses, Ottawa Hospital Research Institute, Ottawa (ON), 2009.

[16] V. N. Nyaga, M. Arbyn, and M. Aerts, "Metaprop: a Stata command to perform meta-analysis of binomial data," Archives of Public Health, vol. 72, p. 39, 2014.

[17] Y. Hayashino, Y. Noguchi, and T. Fukui, "Systematic evaluation and comparison of statistical tests for publication bias," Journal of Epidemiology, vol. 15, pp. 235-243, 2005.

[18] J. P. Souza, C. Pileggi, and J. G. Cecatti, "Assessment of funnel plot asymmetry and publication bias in reproductive health meta-analyses: an analytic survey," Reproductive Health, vol. 4, pp. 1-6, 2007.

[19] S. Brackmann, S. N. Andersen, G. Aamodt et al., "Relationship between clinical parameters and the colitis-colorectal cancer interval in a cohort of patients with colorectal cancer in inflammatory bowel disease," Scandinavian Journal of Gastroenterology, vol. 44, pp. 46-55, 2009.

[20] J. Askling, P. W. Dickman, P. Karlén et al., "Colorectal cancer rates among first-degree relatives of patients with inflammatory bowel disease: a population-based cohort study," Lancet, vol. 357, pp. 262-266, 2001.

[21] J. Tang, O. Sharif, C. Pai, and A. L. Silverman, "Mesalamine protects against colorectal cancer in inflammatory bowel disease," Digestive Diseases and Sciences, vol. 55, pp. 1696-1703, 2010.

[22] B. A. Lashner, K. S. Provencher, D. L. Seidner, A. Knesebeck, and A. Brzezinski, "The effect of folic acid supplementation on the risk for cancer or dysplasia in ulcerative colitis," Gastroenterology, vol. 112, pp. 29-32, 1997.

[23] J. B. Kisiel, E. V. Loftus Jr., S. W. Harmsen, A. R. Zinsmeister, and W. J. Sandborn, "Outcome of sporadic adenomas and adenoma-like dysplasia in patients with ulcerative colitis undergoing polypectomy," Inflammatory Bowel Diseases, vol. 18, pp. 226-235, 2011.

[24] V. Bergeron, A. Vienne, H. Sokol et al., "Risk factors for neoplasia in inflammatory bowel disease patients with pancolitis," The American Journal of Gastroenterology, vol. 105, 2405 pages, 2010.

[25] J. E. Baars, C. W. Looman, E. W. Steyerberg et al., "The risk of inflammatory bowel disease-related colorectal carcinoma is limited: results from a nationwide nested case-control study," Official journal of the American College of Gastroenterology| ACG, vol. 106, p. 319, 2011.

[26] D. T. Rubin, A. Losavio, N. Yadron, D. Huo, and S. B. Hanauer, "Aminosalicylate therapy in the prevention of dysplasia and colorectal cancer in ulcerative colitis," Clinical Gastroenterology and Hepatology, vol. 4, pp. 1346-1350, 2006.

[27] B. A. Lashner, B. D. Shapiro, A. Husain, and J. R. Goldblum, "Evaluation of the usefulness of testing for p53 mutations in colorectal cancer surveillance for ulcerative colitis," The American Journal of Gastroenterology, vol. 94, p. 456, 1999.

[28] X.-R. Wu, F. H. Remzi, X.-L. Liu et al., "Disease course and management strategy of pouch neoplasia in patients with underlying inflammatory bowel diseases," Inflammatory Bowel Diseases, vol. 20, pp. 2073-2082, 2014.

[29] M. Rutter, B. Saunders, K. Wilkinson et al., "Severity of inflammation is a risk factor for colorectal neoplasia in ulcerative colitis," Gastroenterology, vol. 126, pp. 451-459, 2004.

[30] P. Freire, P. Figueiredo, R. Cardoso et al., "Predictive value of rectal aberrant crypt foci for intraepithelial neoplasia in ulcerative colitis-a cross-sectional study," Scandinavian Journal of Gastroenterology, vol. 49, pp. 1219-1229, 2014. 
[31] T. M. Connelly, A. S. Berg, L. R. Harris Iii et al., "Ulcerative colitis neoplasia is not associated with common inflammatory bowel disease single-nucleotide polymorphisms," Surgery, vol. 156, pp. 253-262, 2014.

[32] M. Lutgens, S. Vermeire, M. Van Oijen et al., "Colitis. A rule for determining risk of colorectal cancer in patients with inflammatory bowel disease," Clinical Gastroenterology and Hepatology, vol. 13, pp. 148-154.e1, 2015.

[33] D. T. Rubin, D. Huo, J. A. Kinnucan, M. S. Sedrak, N. E. Mccullom, and A. P. Bunnag, "Raun-Royer EP, Cohen RD, Hanauer SB, Hart J. Inflammation is an independent risk factor for colonic neoplasia in patients with ulcerative colitis: a case-control study," Clinical Gastroenterology and Hepatology, vol. 11, pp. 1601-1608.e4, 2013.

[34] M. Shinozaki, T. Muto, K. Suzuki et al., "Chronic active disease reflects cancer risk in ulcerative colitis," Japanese Journal of Cancer Research, vol. 90, pp. 1066-1070, 1999.

[35] E. Mooiweer, A. E. Van Der Meulen, A. A. Van Bodegraven et al., "Neoplasia yield and colonoscopic workload of surveillance regimes for colorectal cancer in colitis patients: a retrospective study comparing the performance of the updated AGA and BSG guidelines," Inflammatory bowel diseases, vol. 19, pp. 2603-2610, 2013.

[36] F. S. Velayos, E. V. Loftus Jr., T. Jess et al., "Predictive and protective factors associated with colorectal cancer in ulcerative colitis: a case-control study," Gastroenterology, vol. 130, pp. 1941-1949, 2006.

[37] J. Eaden, K. Abrams, A. Ekbom, E. Jackson, and J. Mayberry, "Colorectal cancer prevention in ulcerative colitis: a casecontrol study," Alimentary Pharmacology \& Therapeutics, vol. 14, pp. 145-153, 2000.

[38] S. Bopanna, M. Roy, P. Das et al., "Role of random biopsies in surveillance of dysplasia in ulcerative colitis patients with high risk of colorectal cancer," Intestinal Research, vol. 14, pp. 264269, 2016.

[39] A. Parian, J. Koh, B. N. Limketkai et al., “Association between serrated epithelial changes and colorectal dysplasia in inflammatory bowel disease," Gastrointestinal Endoscopy, vol. 84, pp. 87-95.e1, 2016.

[40] C. Rini, L. Jandorf, H. Valdimarsdottir, K. Brown, and S. H. Itzkowitz, "Distress among inflammatory bowel disease patients at high risk for colorectal cancer: a preliminary investigation of the effects of family history of cancer, disease duration, and perceived social support," Psychooncology, vol. 17, pp. 354-362, 2008.

[41] L. Lakatos, G. Mester, Z. Erdelyi et al., "Risk factors for ulcerative colitis-associated colorectal cancer in a Hungarian cohort of patients with ulcerative colitis: results of a population-based study," Inflammatory Bowel Diseases, vol. 12, pp. 205-211, 2006.

[42] B. A. Lashner, "Red blood cell folate is associated with the development of dysplasia and cancer in ulcerative colitis," Journal of Cancer Research and Clinical Oncology, vol. 119, pp. 549-554, 1993.

[43] L. A. Derikx, W. Kievit, J. P. Drenth et al., "Prior colorectal neoplasia is associated with increased risk of ileoanal pouch neoplasia in patients with inflammatory bowel disease," Gastroenterology, vol. 146, pp. 119-128.e1, 2014.

[44] S. V. Adams, D. J. Ahnen, J. A. Baron et al., "Survival after inflammatory bowel disease-associated colorectal cancer in the Colon Cancer Family Registry," World Journal of Gastroenterology: WJG, vol. 19, p. 3241, 2013.
[45] T. Jess, C. Rungoe, and L. Peyrin-Biroulet, "Risk of colorectal cancer in patients with ulcerative colitis: a meta-analysis of population-based cohort studies," Clinical Gastroenterology and Hepatology, vol. 10, pp. 639-645, 2012.

[46] C. Canavan, K. Abrams, and J. Mayberry, "Meta-analysis: colorectal and small bowel cancer risk in patients with Crohn's disease," Alimentary Pharmacology \& Therapeutics, vol. 23, pp. 1097-1104, 2006.

[47] C. M. Johnson, C. Wei, J. E. Ensor et al., "Meta-analyses of colorectal cancer risk factors," Cancer Causes \& Control, vol. 24, pp. 1207-1222, 2013.

[48] M. W. Lutgens, M. G. Van Oijen, G. J. Van Der Heijden, F. P. Vleggaar, P. D. Siersema, and B. Oldenburg, "Declining risk of colorectal cancer in inflammatory bowel disease: an updated meta-analysis of population-based cohort studies," Inflammatory Bowel Diseases, vol. 19, pp. 789-799, 2013.

[49] R. Shivashankar, W. J. Tremaine, W. S. Harmsen, and E. V. Loftus Jr., "Incidence and prevalence of Crohn's disease and ulcerative colitis in Olmsted County, Minnesota from 1970 through 2010," Clinical Gastroenterology and Hepatology, vol. 15, article 27856364, pp. 857-863, 2017.

[50] J. M. L. Parente, C. S. R. Coy, V. Campelo et al., "Inflammatory bowel disease in an underdeveloped region of Northeastern Brazil," World Journal of Gastroenterology: WJG, vol. 21, p. 1197, 2015.

[51] J. M. Paredes, G. M. Otoya, A. R. P. Mestanza et al., "Epidemiological and clinical characteristics of inflammatory bowel disease in a tertiary referral hospital in Lima-Peru," Revista de Gastroenterología del Perú, vol. 36, pp. 209-218, 2016.

[52] R. Siegel, C. Desantis, and A. Jemal, "Colorectal cancer statistics, 2014," CA: a Cancer Journal for Clinicians, vol. 64, pp. 104-117, 2014.

[53] F. A. Haggar and R. P. Boushey, "Colorectal cancer epidemiology: incidence, mortality, survival, and risk factors," Clinics in Colon and Rectal Surgery, vol. 22, pp. 191-197, 2009.

[54] J. W. Chung, J. J. Park, Y. J. Lim et al., "Gastrointestinal cancer risk in patients with a family history of gastrointestinal cancer," The Korean Journal of Gastroenterology, vol. 71, pp. 338-348, 2018.

[55] L. A. Torre, F. Bray, R. L. Siegel, J. Ferlay, J. Lortet-Tieulent, and A. Jemal, "Global cancer statistics, 2012," CA: a Cancer Journal for Clinicians, vol. 65, pp. 87-108, 2015.

[56] M. Arnold, M. S. Sierra, M. Laversanne, I. Soerjomataram, A. Jemal, and F. Bray, "Global patterns and trends in colorectal cancer incidence and mortality," Gut, vol. 66, pp. 683-691, 2017.

[57] S. C. Ng, H. Y. Shi, N. Hamidi et al., "Worldwide incidence and prevalence of inflammatory bowel disease in the 21st century: a systematic review of population-based studies," Lancet, vol. 390, pp. 2769-2778, 2017.

[58] M. Norouzinia, V. Chaleshi, A. H. M. Alizadeh, and M. R. Zali, "Biomarkers in inflammatory bowel diseases: insight into diagnosis, prognosis and treatment," Gastroenterology and Нераtology From Bed to Bench, vol. 10, p. 155, 2017.

[59] M. Norouzinia and N. Naderi, "Personalized management of IBD; is there any practical approach?," Gastroenterology and Hepatology From Bed to Bench, vol. 8, p. 1, 2015.

[60] J. J. Barendregt, S. A. Doi, Y. Y. Lee, R. E. Norman, and T. Vos, "Meta-analysis of prevalence," Journal of Epidemiology and Community Health, vol. 67, pp. 974-978, 2013. 